\title{
BIOTECNOLOGÍA Y GENÉTICA: EL MAPA DE LA ACREDITACIÓN LA PRIMERA FASE DEL PROCESO DE ACREDITACIÓN ANTE CONEAU
}

\author{
Leandro A. Rossi* \\ Universidad Nacional de Moreno, Argentina \\ leandro.a.rossi@gmail.com
}

Recibido: 5/08/2020 - Aceptado: 10/12/2020

\section{Resumen}

El presente trabajo propone realizar un relevamiento de la acreditación de carreras de Biotecnología y Genética a fin de esbozar un mapa general sobre la primera fase. Para ello se realizó la revisión y análisis de las resoluciones emitidas por la CONEAU sobre cada una de las carreras evaluadas en el marco de la normativa general vigente (Ley de Educación Superior - № 24.521) y la normativa específica establecida por el Ministerio de Educación y la CONEAU.

A los fines del análisis, hemos planteado nuestro trabajo en dos partes sustantivas: por un lado, el marco normativo general y específico que regula los procesos de acreditación de carreras declaradas de interés público y, por otra parte, su aplicación en el caso de las carreras de Biotecnología y Genética a partir de tres ejes de estudio.

Entre los resultados podemos visibilizar cual es la cobertura geográfica de la oferta de carreras, la fortaleza en la función investigación, la alta formación docente y las posibilidades de mejora en extensión.

Palabras clave: Biotecnología - Genética - CONEAU - Evaluación y acreditación - Argentina.

\section{BIOTECHNOLOGY \& GENETICS: THE CERTIFICATION MAP. THE FIRST CERTIFICATION PROCESS CONDUCTED BY CONEAU}

\begin{abstract}
This paper aims to carry out a survey on the certification processes related to the careers of Biotechnology and Genetics in order to outline the general landscape of the first phase.

For this purpose, we have reviewed and analyzed the resolutions issued by CONEAU on each of the evaluated careers within the framework of the current general regulations (Higher Education Law - No. 24 521) and the specific legislation established by the Ministry of Education and CONEAU.

For the purpose of analysis, our work is outlined in two major parts: the general and specific regulatory framework which legislates on the accreditation processes of public interest careers, and their application on Biotechnology and Genetics from a three-axes perspective.

Among the delivered outcomes we can notice the geographical coverage of careers' availability, research capabilities, the highly qualified teaching staff and the possibilities to improve extension.

\footnotetext{
*Docente extraordinario y Miembro del Comité Científico Académico (COCIAC) del Centro de Estudios para el Desarrollo Territorial (CEDET) en Universidad Nacional de Moreno (UNM). 2019/actualidad. Asesor Técnico en Acreditación y Evaluación Universitaria de la Secretaría Académica en la Pontificia Universidad Católica Argentina (UCA). 2018actualidad. Integrante del equipo técnico de la Comisión Nacional de Evaluación y Acreditación Universitaria (CONEAU), Ministerio de Educación. Dirección de Acreditación de Carreras, Área de Acreditación de Carreras de Grado y Posgrado. 2010/2018.
} 
Keywords: Biotechnology - Genetics - CONEAU - Evaluation and certification - Argentina.

\section{Introducción}

Uno de los principales cambios en los sistemas de educación superior, en un escenario signado por el avance del proceso de globalización, fue el foco puesto por los gobiernos en el diseño, implementación y desarrollo de procedimientos de evaluación con un objetivo principal: la calidad de la educación superior (CINDA, 2007; Fernández Lamarra, 2010). En Latinoamérica, si bien se contemplan diversas particularidades y características según cada país, el camino elegido ha sido el de la evaluación a través de organismos creados a partir de los Estados.

Este proceso iniciado a fines de la década de 1980 tuvo en Argentina un impacto concreto a mediados de 1995 cuando se establecieron nuevas pautas para las universidades a partir de la sanción por el Congreso Nacional de la Ley de Educación Superior (LES - Ley № 24.521) ${ }^{1}$. Estas nuevas reglas de juego incrementaron la autonomía universitaria, implementaron nuevos mecanismos de financiamiento y establecieron la evaluación al nivel de programas e instituciones (Claverie, González y Pérez, 2008). De esta manera el gobierno tomó la decisión de incorporarse a los países que empezaron a evaluar la calidad académica de su educación superior (Krotsch, 1994 y 2001) en el contexto de la "segunda reforma del estado" (Ozslak, 1999; Naim, 1995; Banco Mundial 1997 y 1998) y definió ciertos criterios generales básicos: qué evaluar, qué acreditar, cómo y las implicancias de cada proceso.

En el marco del ideario descentralizador, de búsqueda de mayor efectividad del accionar estatal y de desarrollo de un perfil orientado al mercado, es que la LES crea la Comisión Nacional de Evaluación y Acreditación Universitaria (CONEAU) ${ }^{2}$. De acuerdo con Buchbinder (2010), la creación de la CONEAU es resultado directo de las recomendaciones realizadas por el Banco Mundial para mejorar la eficiencia del sistema universitario, en el marco de las exigencias del organismo multilateral para brindar financiamiento al sistema, en un período que puso en debate diversos problemas: acceso, equidad, calidad, acreditación, evaluación y control (Krotsch, 2002; Villanueva, 2006; Buchbinder, 2008; Márquez y Marquina, S/D).

Desde la creación de la CONEAU y su puesta en funcionamiento, se han desarrollado diversos trabajos que abordan los mecanismos de evaluación (Fernández Lamarra, 2002 y 2007; Gómez, 2016; Fernández Lamarra y Aiello, 2014; Aiello, 2017; Araujo, 2007), de acreditación (Fernández Lamarra, 2003), las características del organismo (Isuani, 2003; Ozslak, 2005), la institucionalización de las políticas (Araujo y Trotta, 2011), entre otros abordajes.

Con respecto a las repercusiones que la CONEAU ha tenido en el sistema, se destaca el trabajo de Corengia, en tanto aborda el impacto en las funciones universitarias (docencia, investigación, extensión y gestión) como así también en los actores institucionales (Corengia, 2015). Además, la propia agencia ha desarrollado estudios de impacto para el área de acreditación de carreras de grado, contemplando algunas de las titulaciones acreditadas: Ingeniería Agronómica (CONEAU, 2014), Ingeniería (CONEAU, 2015), Veterinaria (CONEAU, 2016) y Medicina (CONEAU, 2017).

Por lo tanto, este breve trabajo tiene como objetivo realizar un primer estudio exploratorio sobre los procesos de acreditación en dos disciplinas que aún no han sido abordadas (Biotecnología y Genética), a fin de confeccionar un mapa del proceso de acreditación en el marco de la primera fase. Se pretende, además, identificar las características principales de estas disciplinas a partir de la información resultante de la evaluación realizada por CONEAU y, con el objetivo de contextualizar la situación de estas disciplinas al interior del sistema universitario, comparar los datos obtenidos con los resultados de otros procesos de acreditación de primera fase. El trabajo se organiza sobre la base de 3 ejes seleccionados a partir de la estructura de los informes desarrollados por la agencia para todas las titulaciones evaluadas: 1) investigación y vinculación con el medio, 2) cuerpo académico y 3) alumnos y graduados.

Para alcanzar los objetivos propuestos, hemos realizado, en primer lugar, una revisión del marco normativo general (leyes, decretos) y específico (normativa ministerial y de la CONEAU) a fin de determinar con claridad el funcionamiento del proceso y los roles de los actores intervinientes. En segundo lugar, se relevaron y analizaron las resoluciones emitidas por la agencia en el marco de la primera fase de las disciplinas escogidas para el análisis. Por lo tanto, la muestra se compone de la totalidad de resoluciones emitidas hasta el momento en el marco de la primera fase de acreditación de Biotecnología y Genética, en donde se incluyen los recursos de reconsideración. Han quedado por fuera las carreras que aún se encuentran en proceso de evaluación al momento de la escritura del presente trabajo.

\section{Primera Parte El marco normativo general}

La LES estableció un nuevo marco regulatorio para el sistema universitario argentino y, en ese nuevo contexto, definió mecanismos de evaluación y acreditación asignados a la CONEAU, un organismo creado en esa misma normativa, de carácter descentralizado y que funciona desde entonces en jurisdicción del Ministerio de Educación de la Nación ${ }^{3}$.

\footnotetext{
${ }_{1}^{1}$ Promulgada por el Decreto № 268/95 y publicada el 10 de agosto de 1995 (Boletín Oficial № 28.204).

${ }^{2}$ El Decreto $N^{\circ} 173 / 96$ estableció las pautas normativas y reglamentarias mínimas a fin de fijar los aspectos formales para la designación de los integrantes del organismo, su estructura interna básica y algunas pautas fundamentales para su funcionamiento. Fue modificado por el Decreto $N^{\circ} 705 / 97$.

${ }^{3}$ La región cuenta con modelos diversos, por ejemplo, el sistema de educación superior argentino que se caracteriza por el fuerte rol estatal y por contar con una agencia única que además tiene voz ante la eventual creación de organismos
} 
La LES establece que los títulos correspondientes a profesiones reguladas por el Estado, "cuyo ejercicio pudiera comprometer el interés público poniendo en riesgo de modo directo la salud, la seguridad, los derechos, los bienes o la formación de los habitantes” (art. № 43, LES) deberán respetar la carga horaria mínima que fije el Ministerio de Educación en acuerdo con el Consejo de Universidades y, además, deberán ser acreditadas de manera periódica por la CONEAU.

En este escenario, el Consejo de Universidades (CU) mediante el Acuerdo Plenario $N^{\circ} 137 / 15$ y el Ministerio de Educación a través de la Resolución Ministerial № 284/16, decidieron incluir a la titulación de Licenciatura en Biotecnología y de Ingeniería en Biotecnología en el régimen establecido por el artículo $\mathrm{N}^{\circ}$ 43 de la LES. Lo mismo ocurrió para la titulación de Licenciatura en Genética a través del Acuerdo Plenario CU No 138/15 y de la Resolución Ministerial № 244/16.

Como paso subsiguiente, en el Acuerdo Plenario CU No 142/16, el Consejo prestó acuerdo a los contenidos básicos, carga horaria mínima, criterios de intensidad de la formación práctica y las actividades reservadas para quienes cuenten con el título de Licenciado/a en Biotecnología que fueron reflejados en la Resolución MEyD No 1637/17. En el Acuerdo Plenario CU $N^{\circ} 143 / 16$, se prestó acuerdo para las características específicas de la Ingeniería en Biotecnología que fueron avaladas por el Ministerio en la Resolución MEyD N 903/17; y en el Acuerdo Plenario CU N 144/16 se realizó la acción equivalente para la Licenciatura en Genética que el Ministerio plasmó en la Resolución MEyD N 901/17.

Allí se abrió un plazo de 12 meses para que las instituciones universitarias adecuen sus carreras a las nuevas pautas para luego empezar a ser evaluadas por la CONEAU.

\section{El procedimiento: la acreditación de carreras de grado}

La Dirección de Acreditación es el área de CONEAU que tiene dentro de sus competencias las funciones de evaluación y acreditación de carreras y proyectos de carreras de grado, declaradas de "interés público" (art. № 43, LES). Todas estas deben solicitar su acreditación ante el organismo a fin de lograr la validez nacional (Resoluciones Ministeriales $N^{\circ} 6 / 97$ y $N^{\circ} 51 / 10$ ), así como el reconcomiendo oficial del título por parte del Ministerio de Educación Nacional. De acuerdo con el art. $N^{\circ} 44$ de la LES y con el Decreto $N^{\circ}$ 499/95 (modificado por el Decreto N²219/10), las carreras deberán renovar su acreditación cada 3 o 6 años de acuerdo con las evaluaciones del desarrollo alcanzado y el grado de cumplimiento de los estándares.

Los procedimientos actuales para carreras de grado en funcionamiento fueron formalizados en la Ordenanza CONEAU No $63 / 17^{4}$. Alli se establece que el organismo realizará las convocatorias públicas que alcanzan a las carreras incluidas en el art. $N^{\circ} 43$ de la LES, que las instituciones universitarias deberán formalizar las carreras a presentar, a partir del cual se abre un plazo mínimo de 4 meses para el desarrollo de la autoevaluación, que tiene como objetivo que se analice al interior de la institución ${ }^{5}$ el grado de cumplimiento de los estándares y la necesidad de implementar planes de mejora al respecto.

Paralelamente, la CONEAU debe aprobar la nómina de evaluadores (Ordenanza CONEAU N 12/97), instancia donde las instituciones tienen derecho a la recusación fundada de expertos. Luego el organismo procede a conformar una Comisión Asesora disciplinar para posteriormente convocar y conformar los Comités de Pares (Decreto N 173/96), cuya tarea consiste en el análisis de la información presentada por las instituciones, la visita de los espacios utilizados en el proceso educativo ${ }^{6}$, para luego elaborar los informes de evaluación correspondientes.

Como paso previo al inicio del proceso de toma de decisiones, la agencia da vista de los informes de evaluación a fin de que las instituciones puedan completar su presentación. Con esa nueva información, el Comité de Pares elabora, con el asesoramiento del equipo profesional técnico del organismo, un nuevo informe que será elevado para la toma de conocimiento de la Comisión, a los efectos de emitir fundadamente una resolución de acreditación.

La resolución que emita la CONEAU considerará los siguientes posibles resultados:

1. Acreditación por un periodo de 6 años: para las carreras que cumplan con el perfil previsto por los estándares.

2. Acreditación por un periodo de 3 años $^{7}$, para las carreras que ${ }^{8}$ :

de acreditación privados y, como contrapartida, el caso de Chile, con el sistema de educación superior más privatizado de la región y en donde coexisten el Consejo Nacional de Educación, la Comisión Nacional de Acreditación, y agencias privadas (Robledo, 2015)

${ }^{4}$ En noviembre de 2017, al momento de la convocatoria de las carreras de Biotecnología y Genética, aún estaba vigente la Ordenanza CONEAU N58/11, que a finales de 2017 fue reemplazada por la Ordenanza CONEAU N $63 / 17$. El cambio de normativa no alteró cuestiones estructurales del proceso, sino que explicitó mejor algunos detalles procedimentales e incorporó los pasos a seguir referidos a los Trámites a Distancia (TAD) del sistema de Gestión Documental Electrónica (GDE).

${ }^{5}$ El proceso procura la participación de todos los integrantes de la comunidad académica.

' Las carreras que se presentan a acreditación por primera vez o aquellas que, habiéndose presentado previamente, hayan sido no acreditadas, serán visitadas por los pares evaluadores. Para los casos de segunda fase de acreditación o en los casos de un nuevo ciclo de acreditación con la misma Resolución Ministerial de estándares, el organismo evaluará la necesidad de visitar esas carreras nuevamente (Ordenanza CONEAU N 63/17, puntos 7.2 y 7.3).

${ }^{7}$ En la primera fase de carreras de Medicina las carreras acreditaban (por 6 años) o no acreditaban. Posteriormente, se incluyó la posibilidad intermedia de los 3 años, con Ingeniería.

${ }^{8}$ En las resoluciones de acreditación el organismo podrá disponer un seguimiento de las acciones que la institución universitaria debe cumplir para el desarrollo de la carrera (Ordenanza CONEAU N ${ }^{\circ}$ 63/17, punto 8.7). La Ordenanza CONEAU $N^{\circ} 70 / 20$ aprueba las características, procedimientos y alcances de los seguimientos previstos en las 
a- cumplan con el perfil previsto por los estándares, pero no hayan completado un ciclo de dictado.

b- si bien no lograron el perfil previsto, hubiese elementos suficientes para considerar que la carrera desarrolla efectivamente planes de mejora cuyo impacto permitirá lograr el perfil mencionado previamente en un plazo razonable. Al vencimiento del plazo, se inicia la segunda fase del proceso para evaluar la posibilidad de la extensión de la acreditación por 3 años más.

3. No acreditación: para aquellas carreras que no cumplan con el perfil previsto y cuyos planes de mejora sean considerados no factibles e insuficientes para poder alcanzarlo (Ordenanza CONEAU N 63/17).

El último acto administrativo del proceso consiste en la instancia de reconsideración de la resolución de no acreditación. Las carreras que no hayan obtenido la acreditación pueden solicitar una ampliación del plazo (hasta un máximo de 6 meses) para realizar las acciones necesarias con el objetivo de subsanar las objeciones que motivaron la no acreditación. Este proceso culmina con una nueva resolución del organismo haciendo lugar al pedido (es decir, otorgando la acreditación o extensión de acreditación) o no haciendo lugar al recurso (sosteniendo la no acreditación).

Este esquema de resultados y la introducción de la posibilidad de que las instituciones desarrollen planes de mejora para subsanar las "debilidades" observadas y alcanzar la acreditación por 3 años, constituye parte de un amplio debate sobre categorizar carreras o impactar en ellas, es decir, sobre la posibilidad de "incluir funciones formativas en procesos de evaluación y control" (Fernández Lamarra y Aiello, 2014, p.33).

\section{Segunda Parte}

\section{La primera fase de acreditación: Biotecnología y Genética}

El jueves 16 de noviembre de 2017 la CONEAU publicó la RESFC-2017-494-APN-CONEAU\#ME donde se convocó a las instituciones universitarias que dictan carreras de Licenciatura en Genética y la RESFC-2017-495-APN-CONEAU\#ME que convocó a las carreras de Licenciatura en Biotecnología y de Ingeniería en Biotecnología a participar del proceso de acreditación ${ }^{9}$. Ambas resoluciones establecieron un cronograma unificado para las dos convocatorias y fijaron una fecha límite para la presentación de las autoevaluaciones: el 30 de mayo de 2018. En ese momento todas las universidades del país que formalizaron sus carreras de Biotecnología y Genética debían presentar la información para ser evaluadas, utilizando los instrumentos y herramientas de recolección de información brindados previamente por CONEAU ${ }^{10}$.

Mediante el Acta № 481/18 la CONEAU aprobó la nómina de pares evaluadores de donde el organismo seleccionó un total de 16 evaluadores para conformar los Comités de Pares ${ }^{11}$ para evaluar a las 17 carreras presentadas a la convocatoria conjunta: 3 Licenciaturas en Genética (Universidad Nacional de Misiones -UNAM-, Universidad Nacional del Noroeste de la Provincia de Buenos Aires -UNNOBA- y Universidad de Morón -UM-), una Ingeniería en Biotecnología (Universidad Nacional de Río Negro -UNRN) y 13 Licenciaturas en Biotecnología (Universidad Nacional de Tucumán - UNT-, Universidad Nacional de Santiago del Estero - UNSE-, Universidad Nacional de Chaco Austral -UNCAUS-, Universidad Nacional de San Luis -UNSL-, Universidad Nacional de Córdoba -UNC-, Universidad Nacional de Rosario -UNR-, Universidad Nacional del Litoral -UNL-, Universidad Nacional de La Plata -UNLP-, Universidad Nacional de Quilmes -UNQ-, Universidad Nacional de San Martín -UNSAM-, Universidad Nacional de Moreno -UNM-, Universidad Argentina de la Empresa -UADE- y Universidad de Morón -UM-). A simple vista se observa que la oferta de carreras está concentrada principalmente en instituciones de gestión pública: 12 carreras de Biotecnología de un total de 14 y 2 de Genética de un total de 3 carreras.

Ordenanzas № ${ }^{\circ}$, № 63, № 64 y № 65. Esta nueva Ordenanza genera un cambio relevante en los procesos de acreditación.

${ }^{9}$ La RESFC-2017-570-APN-CONEAU\#ME, con fecha del 18 de diciembre de 2017, convoca a las carreras de Licenciatura en Biotecnología y de Ingeniería en Biotecnología que tengan entre el $30 \%$ y el $100 \%$ de su carga horaria bajo la opción pedagógica de Educación a Distancia, previa validación del Sistema Institucional de Educación a Distancia (SIED) en el marco de la Resolución ME № 2641/17. La RESFC-2017-569-APN-CONEAU\#ME hace lo propio con las carreras de Licenciatura en Genética a distancia. Sin embargo, no se presentaron carreras bajo esta modalidad de dictado para ser evaluadas en el marco de las mencionadas convocatorias.

${ }^{10}$ El Taller de Presentación de la Guía de Autoevaluación fue realizado el 6 de diciembre de 2017, donde se explicitó la normativa que regula el proceso y las herramientas para la recolección y sistematización de la información de las carreras (Instructivo CONEAU Global).

${ }^{11}$ Los comités se constituyen a partir de las recomendaciones de la Comisión Asesora en base al registro de expertos de la CONEAU conformado a partir de las propuestas de las instituciones universitarias. Los pares deben reunir determinadas condiciones: 1) idoneidad profesional, 2) experiencia académica, 3) capacidad como evaluador y 4) experiencia en conducción, administración y gestión de programas académicos. Además, al momento de ser convocados por CONEAU no pueden estar ejerciendo ningún cargo de gestión. 


\section{Carreras de Biotecnología y Genética en Argentina (CPRES)*}

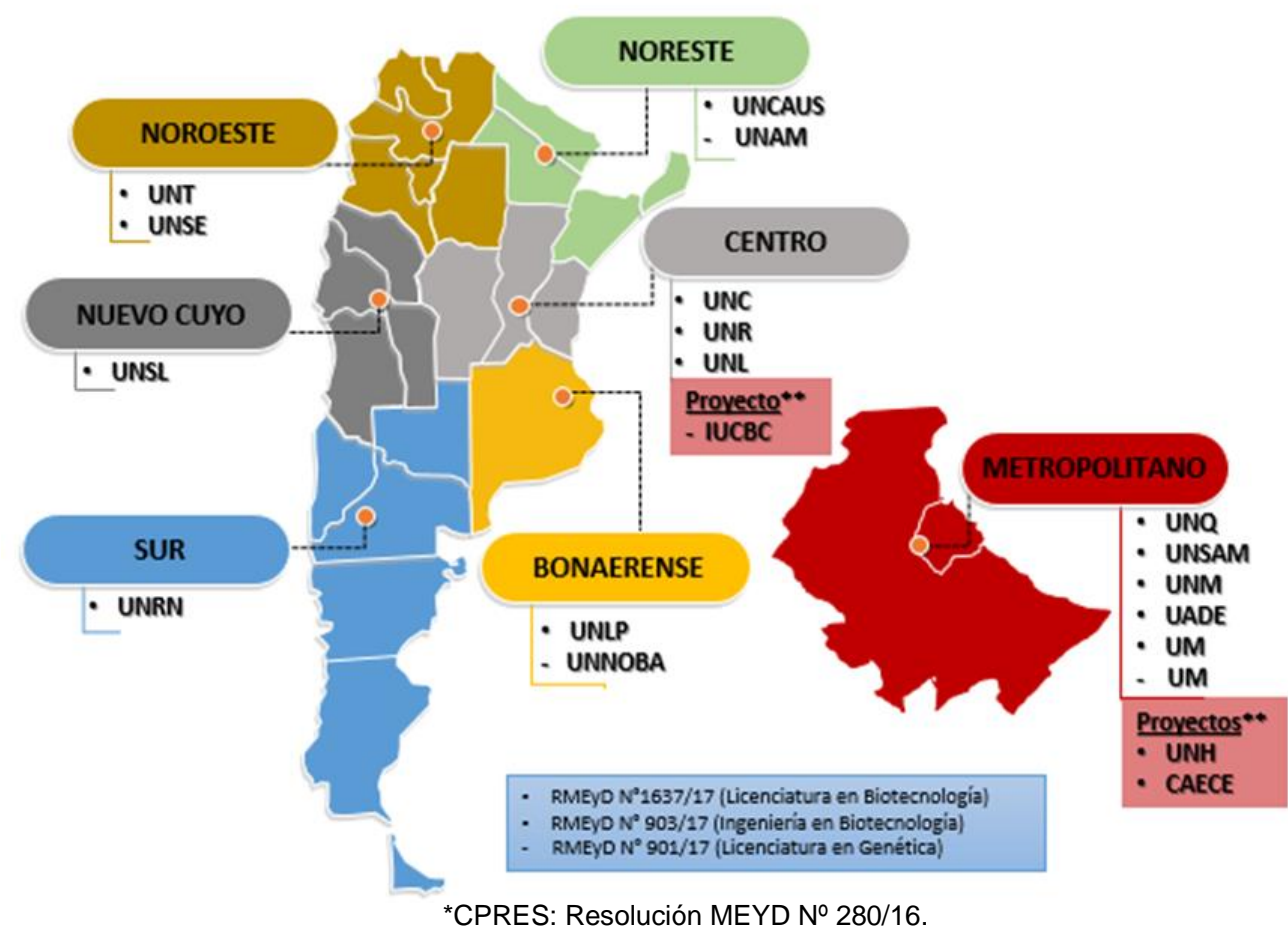

${ }^{*}$ Se incluyen las carreras presentadas en la primera fase de acreditación (2017) y las carreras presentadas como proyecto de carreras de Biotecnología hasta el momento: UNH y CAECE (ambas en 2018) y IUCBC de Genética (abril 2020). No se incluye Ingeniería en Agrobiotecnología (UNSAM).

En el período comprendido entre la presentación realizada por las carreras y fines de septiembre se realizaron las visitas de los evaluadores y el equipo técnico profesional a las instituciones. Entre el 9 y 11 de octubre de 2018 se realizó la reunión de consistencia, última instancia en la que se reúnen todos los pares evaluadores con el objetivo de hacer una puesta en común, consolidar criterios de evaluación finales con respecto a la aplicación de los estándares y debatir cuestiones complejas surgidas durante las visitas que requieran análisis específicos. El paso subsiguiente es la redacción de los Informes de Evaluación, que fueron enviados durante fines de $2018^{12}$.

EI mapa refleja que todos los Consejos Regionales de Planificación de la Educación Superior (CPRES) cuentan con al menos una carrera de Biotecnología, con una mayor concentración de carreras en el CPRES Metropolitano ( $36 \%$ de las 14 carreras presentadas en primera fase). Por otra parte, sólo los CPRES Bonaerense, Metropolitano y Noreste cuentan actualmente con carreras de Genética. De las 17 carreras presentadas, 14 (82\%) corresponden a universidades de gestión pública, y las 3 correspondientes a universidades de gestión privada se encuentran en el CPRES Metropolitano, siendo 2 de esas carreras pertenecientes a la Universidad de Morón.

Luego del envío de Informes de Evaluación y de las respuestas a esos informes por parte de las universidades (Respuestas a la Vista), la CONEAU elaboró 17 resoluciones, de las cuales 12 fueron acreditaciones ( 9 carreras obtuvieron la acreditación por 6 años y 3 lograron acreditar por 3 años) y 5 fueron resoluciones de no acreditación. Estas últimas utilizaron el recurso de reconsideración que prevé la Ordenanza CONEAU N ${ }^{\circ}$ 63/17. En este marco, 3 carreras lograron la acreditación (una por 6 años y 2 por 3 años) mientras que las 2 carreras restantes aún se encuentran en proceso de evaluación (una Licenciatura en Biotecnología y una Licenciatura en Genética).

${ }^{12}$ A la par de la primera fase de acreditación, en el ingreso de carreras nuevas de abril de 2018, se presentaron ante CONEAU 2 nuevos proyectos de carrera de Licenciatura en Biotecnología: la Universidad Nacional de Hurlingham (UNH) y la Universidad de la Cámara Argentina de Comercio y Servicios (CAECE). En abril de 2020 se presentó un proyecto de Licenciatura en Genética del Instituto Universitario de Ciencias Biomédicas de Córdoba (IUCBC). Estos casos no se abordarán en el presente trabajo dado que nuestro objeto es la primera fase de acreditación. Se incluyen en el gráfico $\mathrm{N}^{\circ} 1$ a modo ilustrativo. 
Gráfico N²: Los resultados

Primera Fase - Biotecnología y Genética

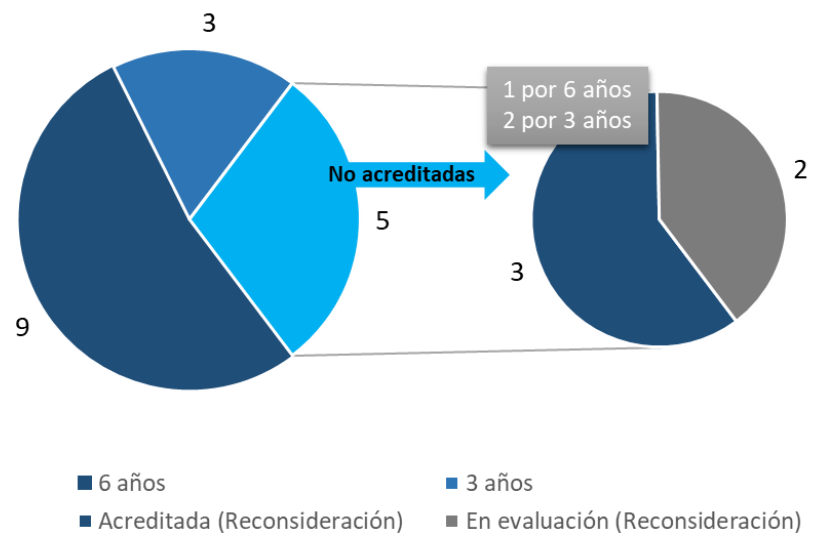

Fuente: Resoluciones CONEAU. Elaboración propia.

Por el momento, el $88 \%$ de las carreras presentadas en las convocatorias analizadas han logrado cumplir con los estándares previstos en las Resoluciones Ministeriales $N^{\circ}$ 901/17, $N^{\circ}$ 903/17 y $N^{\circ}$ 1637/17 o han desarrollado estrategias factibles, viables y concretas a fin de subsanar las debilidades observadas en el corto plazo en el marco de planes de mejoras.

\section{Contexto Institucional}

La primera carrera de Licenciatura en Genética, no sólo de la Argentina sino también de América del Sur, fue creada por la UNAM en el ámbito de la Facultad de Humanidades y Ciencias Sociales, hasta que en 1985 se trasladó a la Facultad de Ciencias Exactas, Químicas y Naturales. Por su parte, la UNR creó en 1989 la primera carrera de Licenciatura en Biotecnología al interior de la Facultad de Ciencias Bioquímicas y Farmacéuticas ${ }^{13}$, como una carrera independiente de la Licenciatura en Química (1985). Por su parte, la única carrera de Ingeniería en Biotecnología del país se dicta en la UNRN, fue creada en 2009 e inició su dictado en 2010 en la Sede Alto Valle - Valle Medio (Villa Regina). De las restantes carreras de ambas disciplinas, la mayoría se crearon a partir del año 2000 (10 carreras) y algunas durante la última década del siglo XX (4 carreras).

Sólo 5 carreras cuentan con un solo plan de estudios vigente, que en todos los casos ha sido modificado al menos una vez, en general en el marco de la primera fase, mientras que 11 carreras han desarrollado un nuevo plan de estudios entre 2017 y 2019 a fin de adecuarse a las Resoluciones Ministeriales.

\section{Investigación y vinculación con el medio}

Un elemento distintivo de estas carreras es su fuerte impronta y perfil para el desarrollo de actividades de investigación. Se desarrollaban al momento del proceso de acreditación un total de 333 proyectos evaluados favorablemente por los pares evaluadores, en donde participaron un total de 596 docentes-investigadores con una participación total de 312 estudiantes $^{14}$. De las 15 carreras acreditadas, 13 cuentan con resultados de estas actividades (publicaciones con y sin referato, presentaciones a congresos, libros, capítulos de libros, etc.), mientras que las 2 carreras restantes han desarrollado planes de mejora para el área de investigación.

\footnotetext{
${ }^{13}$ La Facultad de Ciencias Bioquímicas fue creada en 1970, contaba con las carreras de Farmacia (1924) y Bioquímica (1956), y que luego se denominaría en reconocimiento a ambas carreras, Facultad de Ciencias Bioquímicas y Farmacéuticas. En 1981, en el contexto de la dictadura cívico militar iniciada el 24 de marzo de 1976, la Facultad pasó a denominarse Facultad de Ciencias Básicas. Fue recién en el marco del restablecimiento de la democracia que en 1984 pasó a tener el nombre actual.

${ }^{14}$ Las cifras de estudiantes corresponden a 2017, último año informado.
} 
Gráfico N³: Actividades de investigación.

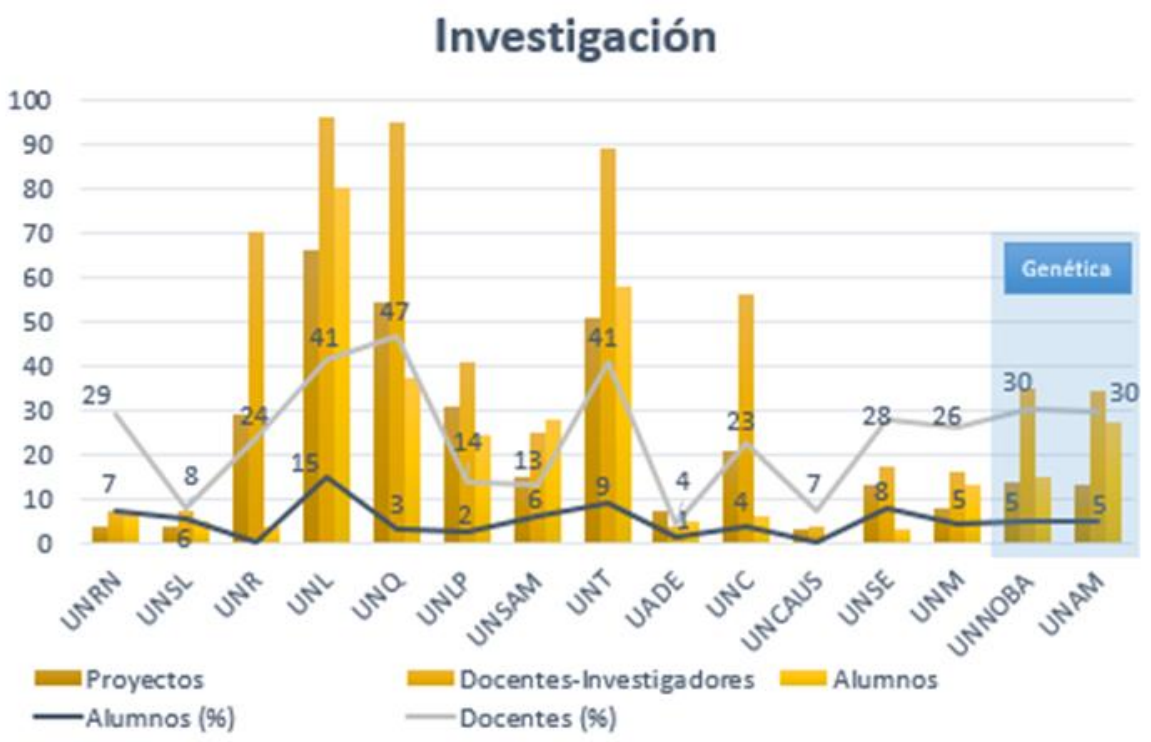

Fuente: Resoluciones CONEAU. Elaboración propia.

*No se incluyen las 2 carreras que aún se encuentran en proceso de evaluación. Tampoco se incluyen proyectos incluidos en el marco de planes de mejora.

**El \% de alumnos está calculado a partir de la cantidad de alumnos participantes en los proyectos en relación con la cantidad total de alumnos de cada carrera durante el último año informado en el proceso de acreditación (2017).

Las carreras de Biotecnología muestran una sólida estructura para el desarrollo de actividades de investigación sobre la base de: 1) cuerpos docentes altamente formados, 2) asignación de dedicaciones para investigación, 3) elevado número de docentes categorizados (Programa de Incentivos y CONICET) y 4) una buena participación de estudiantes en este tipo de actividades (temprana iniciación en investigación). En el ámbito de una ciencia multidisciplinar como la Biotecnología, en donde interactúan las ciencias químicas, biológicas, la física y la informática, la articulación de estos elementos tiene como corolario una gran producción científica, teniendo en cuenta que se desarrolla en el marco de carreras de grado.

Al analizar en detalle cada caso, vemos que las 7 carreras con mayor desarrollo en investigación logran una adecuada sinergia entre estos factores: UNL informó 66 proyectos vigentes, UNQ 54, UNT 51, UNLP 31, UNR 29, UNC 21 y UNSAM 15; todas informaron una buena participación de docentes en los proyectos (entre un $13 \%$ y un $47 \%$ de sus respectivos cuerpos docentes), la gran mayoría de ellos con una destacada formación (entre un $74 \%$ y un $97 \%$ de los docentes investigadores alcanzaron la titulación de doctorado) y con dedicaciones exclusivas. El panorama es similar en Genética donde la participación docente es del $30 \%$. 
Gráfico $\mathbf{N}^{\circ}$ 4: Dedicaciones y categorizaciones en cuerpos docentes.

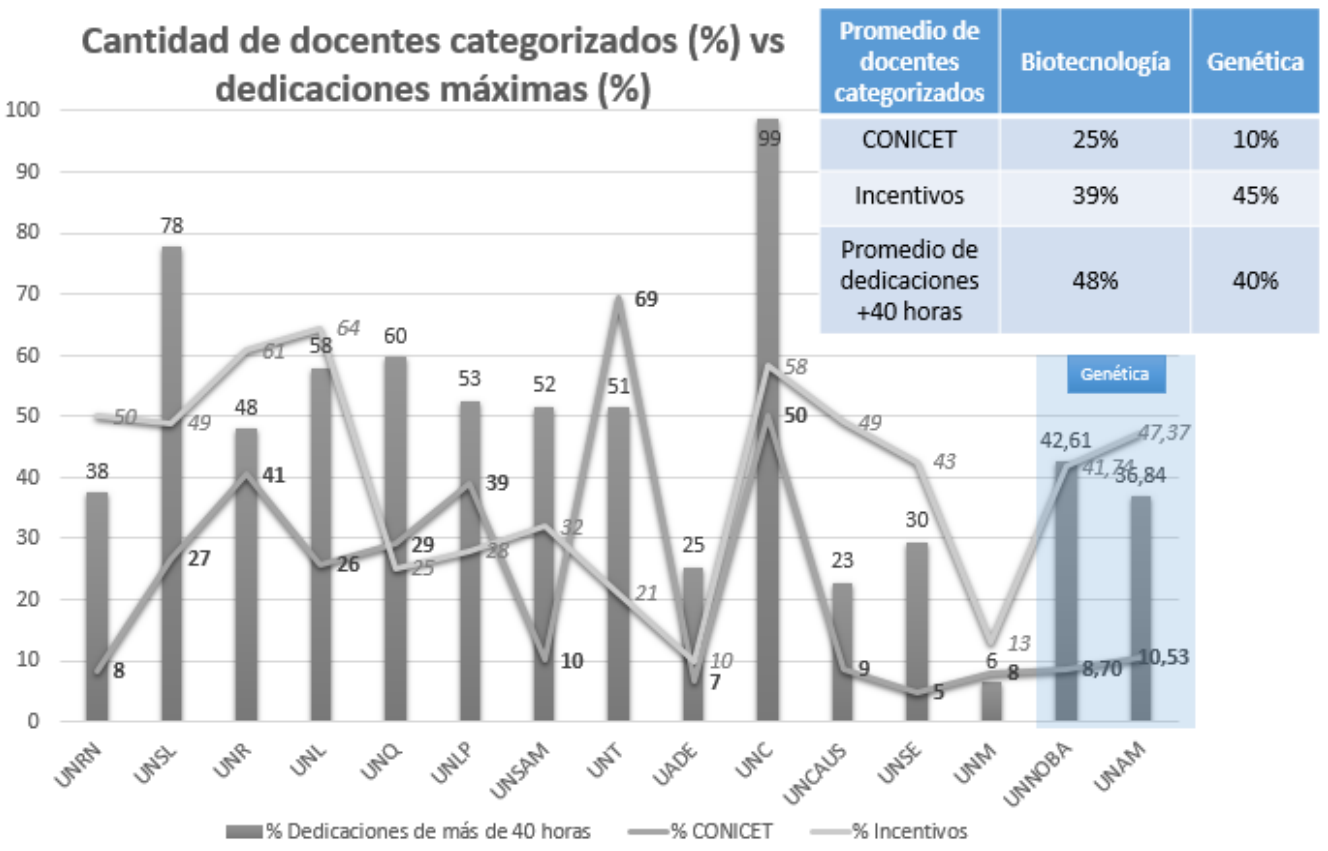

Fuente: Resoluciones CONEAU. Elaboración propia.

*No se incluyen las 2 carreras que aún se encuentran en proceso de evaluación.

Si bien ciertos indicadores de la función investigación que se encontraban en los primeros estándares se han empezado a erosionar o se incluyen en indicadores más amplios en una suerte de transición de criterios ideales a otros más cercanos a la realidad de las carreras (Fernández Lamarra y Aiello, 2014), esto no impacta en nuestras disciplinas de estudio, en tanto se advierte que ambas responden de manera satisfactoria a las pautas establecidas por las normativas ministeriales y constituyen a este tipo de actividades en un elemento distintivo.

Para dimensionar la solidez de la función investigación en estas disciplinas, resulta ilustrativo comparar con las primeras fases de otras disciplinas evaluadas anteriormente: en el caso de las Ingenierías, 196 carreras (un $71 \%$ ) tuvieron compromisos con respecto a la función de investigación y 115 de ellas (59\%) también tuvieron como compromiso incrementar dedicaciones para desarrollar este tipo de actividades (CONEAU, 2015, p.48). En Medicina, 17 carreras (71\%) no tenían un desarrollo adecuado de actividades de investigación en la primera fase y tuvieron que desarrollar planes de mejora para acceder a la acreditación (CONEAU, 2017, p.45). En nuestros casos de estudio, de las 14 carreras de Biotecnología sólo $3(21 \%)$ tuvieron observaciones sobre la función investigación, mientras que en Genética sólo 1 carrera $(33 \%)^{15}$

Si las actividades de investigación constituyen un pilar central en el perfil tanto de las carreras de Biotecnología como de Genética, no se advierte la misma situación con respecto a las actividades de vinculación con el medio y extensión. Considerando el universo de 17 carreras correspondiente a ambas disciplinas, se advierte que en 13 Informes de Evaluación los pares evaluadores hicieron requerimientos relacionados con las actividades de extensión y vinculación con el medio y en un caso se realizó una recomendación para fortalecer el desarrollo de este tipo de actividades, destacando la importancia de que exista un impacto de los saberes propios de la disciplina en la comunidad en donde la carrera se encuentra inserta (RESFC-2019-533-APN-CONEAU\#MECCYT, Recomendación №3). De las 13 carreras en que este aspecto fue observado, en 3 casos el requerimiento fue subsanado en la Respuesta a la Vista, en otros 3 casos fue subsanado, pero igualmente los evaluadores realizaron una recomendación sobre este aspecto, en 5 casos se presentaron planes de mejoras que devinieron en compromisos y los 2 casos restantes corresponden a las carreras que se encuentran en evaluación (reconsideración de no acreditación).

De las carreras acreditadas, 15 cuentan con menos de 10 proyectos o actividades de vinculación con el medio y sólo 2 se destacan con una mayor cantidad de proyectos en desarrollo: UNQ con 19 y UNT con 23 proyectos. Estos datos reflejan que la función de extensión y vinculación con el medio, a través del desarrollo de actividades, programas y proyectos donde las disciplinas en cuestión logren un impacto concreto en beneficio de la comunidad, es un ámbito donde las carreras de Biotecnología y Genética tienen espacio para crecer y desarrollarse.

\footnotetext{
${ }^{15}$ De los 4 casos donde la función investigación fue observada, en 2 casos se generaron compromisos a partir de los planes de mejora presentados y los casos restantes se encuentran en evaluación.
} 


\section{Cuerpo académico}

Los análisis de los perfiles docentes reflejan de forma directa la fortaleza de la función de investigación en estas carreras. En este sentido, el análisis del cuerpo docente puede abordarse desde 3 variables diferentes: 1) la formación, 2) las dedicaciones y 3) la estructura de cargos. Por otra parte, y más allá de los enfoques propuestos para estudiar a los planteles docentes, podemos ver ciertas diferencias disciplinares. Por lo tanto, dividiremos la argumentación en dos cuando sea pertinente. Es importante tener presente en la lectura de los datos que la base de carreras acreditadas de Biotecnología (13) es mayor a Genética (2).

Con respecto a la formación, se advierte que tanto las carreras de Biotecnología como de Genética que se presentaron al primer proceso de acreditación cuentan con cuerpos docentes muy calificados, con destacadas trayectorias y con un perfil de claro corte académico y científico. Tomando el promedio de las carreras de Biotecnología acreditadas, vemos que un $69 \%$ de los docentes cuentan con formación de posgrado, con una muy fuerte preeminencia de doctores (56\% del total de docentes de la carrera). En las carreras de Genética que obtuvieron la acreditación ante CONEAU, la cantidad de docentes posgraduados si bien es menor alcanza a un 53\% de los docentes. En esta disciplina también la mayoría ha obtenido la titulación de doctorado (32\%), pero hay un mayor equilibrio con respecto a las restantes titulaciones de posgrado ( $8 \%$ de especialistas y $13 \%$ de magísteres). Este nivel de formación tiene correlato con la inserción de docentes en el sistema formal de investigación (categorizaciones) que se alinea con altas dedicaciones y terminan por reflejar su impacto en los proyectos de investigación desarrollados en el seno de cada carrera de grado (Gráfico № 4).

Al tomar como referencia los datos que surgen de los procesos de acreditación de otras disciplinas es posible confirmar la fortaleza de Biotecnología y Genética en esta dimensión. El análisis de los datos de la primera fase de Ingeniería Agronómica muestra que un promedio del $43 \%$ de los docentes contaban con formación de posgrado en 2005, de los cuales un 11\% eran doctores (CONEAU, 2014, p.56). Algo similar vemos en la primera fase de acreditación de Veterinaria, donde los posgraduados constituían, en promedio, un $39 \%$ en 2007 , de los cuales $14 \%$ eran doctores (CONEAU, 2016, p.50).

Al volver sobre el esquema de dedicaciones, se advierte que ambas disciplinas asignan altas dedicaciones a sus planteles docentes. Si bien en el promedio de carreras acreditadas en ambos casos se destaca que la mayoría de los docentes cuentan con dedicaciones de más de 40 horas semanales (casi un $48 \%$ en Biotecnología y cerca del $40 \%$ en Genética), la distribución es algo más pareja en Biotecnología (casi el 12\% tiene menos de 9 horas de dedicación, el 24\% se encuentra en el rango 10-19 horas, 13\% entre $20-29$ horas y casi el $4 \%$ entre 30-39\%) mientras que en Genética el segundo mayor conglomerado de dedicaciones se concentra en el rango entre 10-19 horas (casi 35\%), seguido luego por las restantes (15\% entre $20-29$ horas, casi $7 \%$ entre $30-39$ horas y aproximadamente el $4 \%$ con menos de 9 horas).

Gráficos $N^{\circ} 5$ y 6: Cuerpos docentes de Genética y Biotecnología

$$
\begin{gathered}
\text { Cuerpo docente - Genética (Promedio) } \\
\text { - Formación = Dedicaciones = Cargos }
\end{gathered}
$$
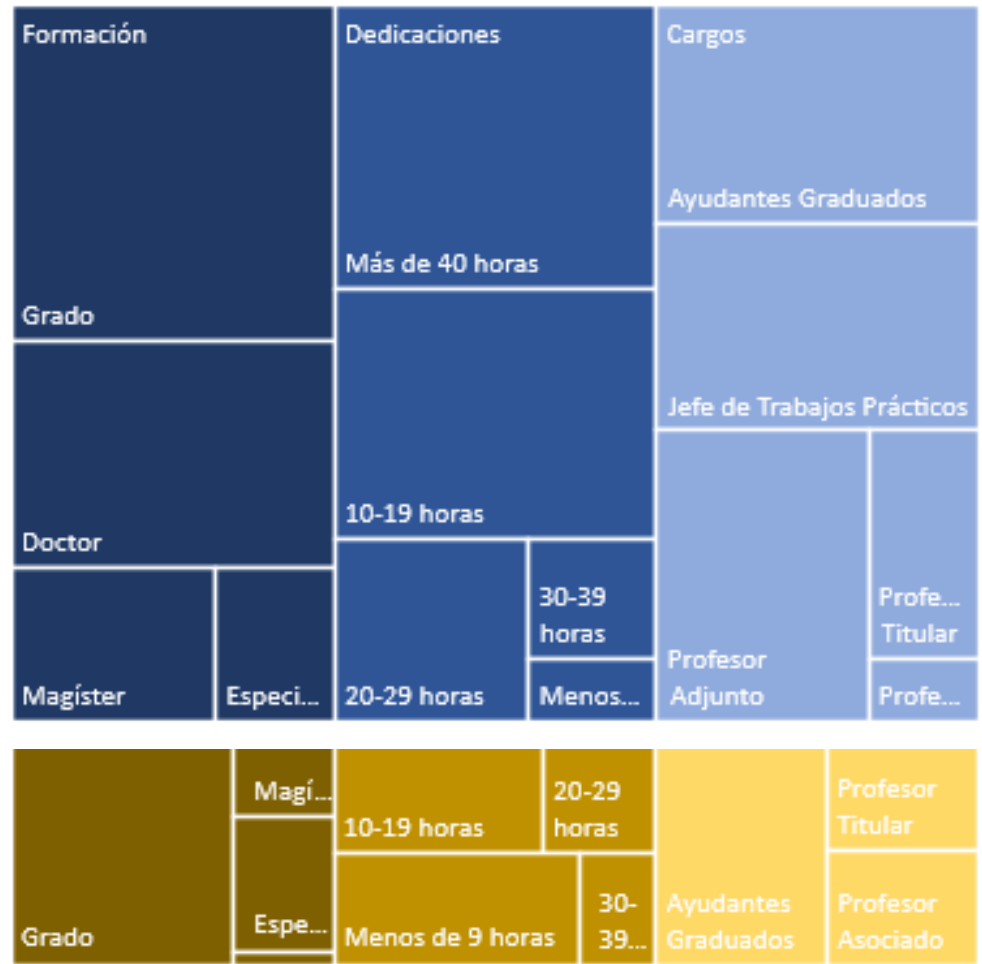
Por otra parte, encontramos estructuras de cargos similares, con cierta forma piramidal, pero con algunas diferencias con respecto a las bases. En el promedio de carreras acreditadas de Biotecnología vemos que un $49 \%$ del cuerpo docente tiene un cargo de Profesor (9\% Titular, $7 \%$ Asociado y $32 \%$ Adjunto), mientras que en Genética es un 40\% (11\% Titular, 3\% Asociado y $26 \%$ Adjunto). Estas mínimas diferencias entre los cargos de Profesores contrastan con mayores diferencias en la base de la estructura de cargos, donde en Biotecnología se observa un 19\% de Ayudantes Graduados mientras que en Genética alcanza a casi un $31 \%$ del cuerpo docente promedio. En ambas disciplinas el cargo de Jefe de Trabajos Prácticos ocupa cerca de un tercio del cuerpo docente y constituye una pieza fundamental en el desarrollo de las actividades prácticas (un $32 \%$ en Biotecnología y un $29 \%$ en Genética).

Al seleccionar variables que siguen la perspectiva de análisis trazada por el Ministerio y la agencia evaluadora, logramos analizar la situación de las carreras de Biotecnología y Genética en un lenguaje común que nos brinda la posibilidad de realizar determinadas comparaciones con otras disciplinas ya acreditadas que cuentan con datos consolidados (CONEAU, 2014, 2015, 2016 y 2017). En primer lugar, esto nos permite visualizar la importancia de la mirada técnica-normativa de la agencia en todo el proceso (Fernández Lamarra y Aiello, 2014), con la distinción entre criterios disciplinares y criterios generales más vinculados a la gestión académica, donde se refleja el perfil del modelo burocrático liderado por CONEAU.

En este sentido, advertimos que la "segunda ola de reformas" con su propuesta de actualización de las capacidades regulatorias del Estado (Naim, 1995) plantea la proliferación de una nueva burocracia: la de los objetivos, metas y resultados (Fisher, 2018). La búsqueda de la eficiencia del sistema (mayor cantidad de graduados, menor desgranamiento, mayor producción científica, etc.) en línea con un concepto de calidad vinculado al "producto" (Márquez y Marquina, S/D, p.19) que requiere de la cuantificación, de la medición y de la autoevaluación continua por parte de las instituciones universitarias y de la evaluación periódica de la agencia, siempre se balancea frente al problema de la "primacía de la evaluación de los símbolos del desempeño sobre el desempeño real” (Fisher, 2018, p.76). Este desafío configura un límite que no podemos abordar en el presente trabajo, en tanto excede a los objetivos que nos hemos planteado, pero constituye una perspectiva de análisis a la que resulta interesante volver una y otra vez a medida que nuevas disciplinas se incorporan a los procesos de acreditación.

En segundo lugar, vemos que esta perspectiva técnico-normativa se transparenta en los resultados de las distintas disciplinas de acuerdo con cómo se ajustan a la metodología e indicadores de evaluación propuestos. En este sentido, pareciera que las disciplinas más académicas y científicas se adaptan mejor al modelo de evaluación (Biotecnología logró que el 50\% acredite por 6 años y Genética un 66\%) mientras que otras disciplinas más profesionales, como surge de nuestros análisis comparativos previos, tuvieron más inconvenientes con respecto a dimensiones tales como investigación, formación docente y dedicaciones (por ejemplo, en Ingeniería Agronómica un 22\% logró acreditar por 6 años, entre las Ingenierías un $11 \%$ y un $7 \%$ en Veterinaria $)^{16}$.

\section{Alumnos y graduados}

Dado que las carreras tuvieron que presentar sus autoevaluaciones a CONEAU en mayo de 2018, la información correspondiente a los estudiantes alcanza hasta el año 2017. De acuerdo con el Departamento de Información Universitaria (DIU) de la Secretaría de Políticas Universitarias (SPU), en 2017 el sistema universitario contaba con un total de 2.005.152 alumnos de pregrado y grado, de los cuales 1.584.392 corresponden a instituciones de gestión estatal y 420.760 a instituciones de gestión privada (Síntesis de información de estadísticas universitarias, 2015-2016 y 2016-2017). En 2017, la totalidad de estudiantes en carreras de Biotecnología era de 5774 ( $0.28 \%$ del total de estudiantes del país), de los cuales $5380(93.17 \%)$ corresponden a instituciones públicas y $394(6.82 \%)$ a instituciones de gestión privada, mientras que las carreras de Genética contaban con 938 alumnos en total (0.04\%), 875 (93.28\%) en universidades de gestión pública y $63(6.71 \%)$ en instituciones de gestión privada.

\footnotetext{
${ }^{16}$ Datos correspondientes a las primeras fases de cada disciplina sin contemplar los resultados de los Recursos de
} Reconsideración. 
Gráfico N 7: Alumnos Genética y Biotecnología (2017).

Genética

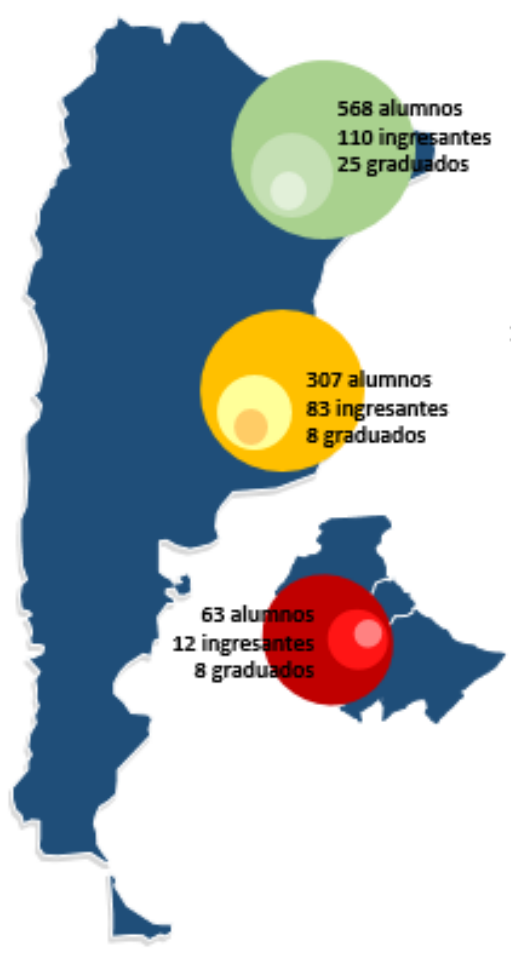

Biotecnología

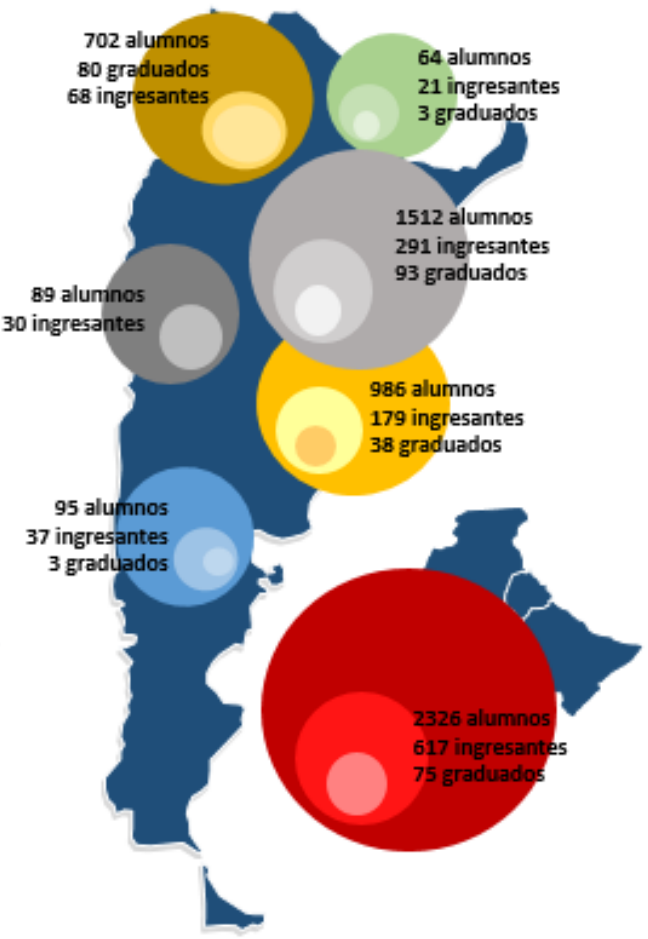

Fuente: CONEAU.Elaboración propia sobre la base de los CPRES.

La Licenciatura en Genética de la UNAM, la primera del país, es la carrera con mayor cantidad de estudiantes de la disciplina, mientras que en Biotecnología la mayor cantidad de estudiantes se concentra en el CPRES Metropolitano, que comprende un total de 5 carreras (3 de gestión pública, con 1932 estudiantes, y 2 de gestión privada que tenían 394 alumnos). El CPRES Centro cuenta con 2 carreras con una importante cantidad de estudiantes de Biotecnología (UNR con 811 alumnos y UNL con 545 estudiantes) mientras que el CPRES Bonaerense tiene a la UNLP con 986 alumnos y, en el Noroeste, la UNT cuenta con 664 alumnos.

La situación es similar por carrera y CPRES con respecto a los ingresantes y egresados. Se destaca que Biotecnología en la UNM, creada en 2016, ha tenido una gran cantidad de ingresantes en los 2 años informados (186 en 2016 y 227 en 2017), siendo una de las pocas carreras biotecnológicas que incrementó la cantidad de ingresantes. En Genética, sólo la UNNOBA presenta un crecimiento continuo de ingresantes en el período 2015-2017 (68, 74 y 83 respectivamente) y de alumnos (261, 292 y 307).

Al considerar los datos de estudiantes de la DIU para la serie 2010-2018 (Gráficos N 8 y 9 ) vemos carreras con una fuerte disminución de estudiantes (Biotecnología de UNT y UNLP) y otras con una caída más suave (UNR, UNL o Genética de UM). Como contrapartida, se ve un fuerte crecimiento en las carreras creadas durante los últimos años y que, al momento de la publicación de las resoluciones de acreditación, aún no contaban con graduados como UNSL (2012), UNC (se creó en 2013 pero inició en 2016), UNSE (2015) y UNM (2016). Tal vez ambos fenómenos estén vinculados y, entre otros factores, la nueva oferta esté produciendo migración de estudiantes al interior de cada CPRES (noroeste, noreste, centro) o entre CPRES cercanos (bonaerense-metropolitano). 
Gráficos N 8 y 9: Carreras de Biotecnología y Genética según crecimiento y disminución de estudiantes.

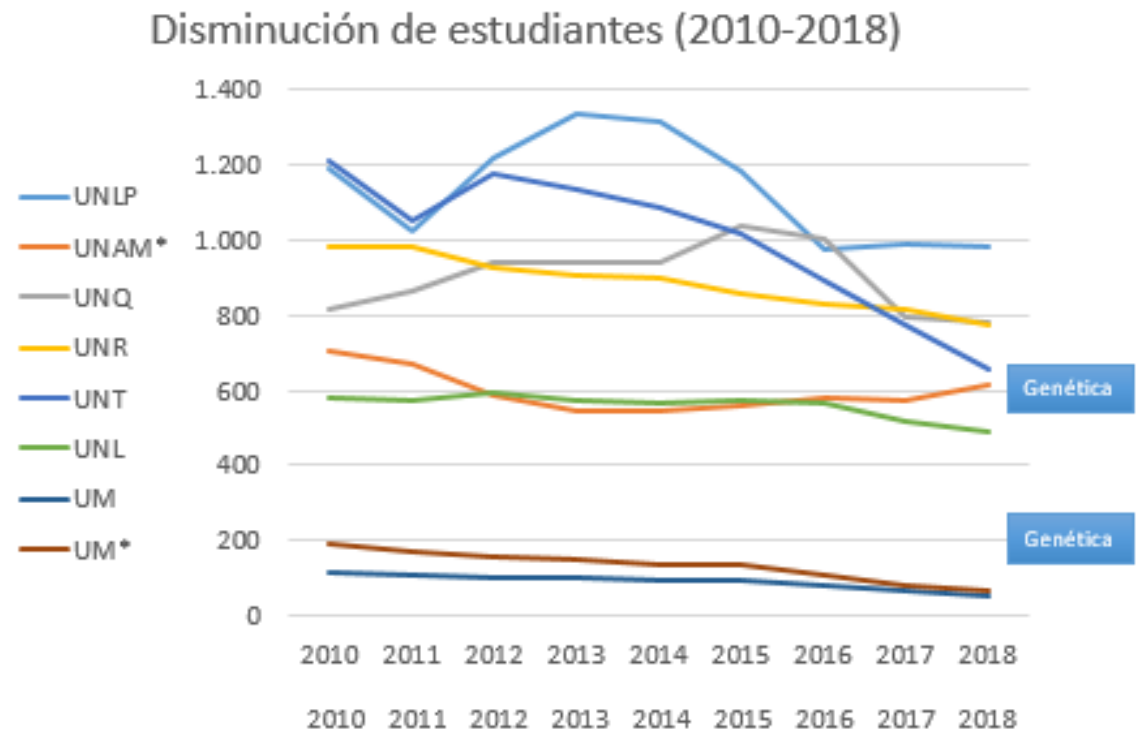

Fuente: SPU - Departamento de Información Universitaria (2020). Elaboración propia. ${ }^{*}$ Carreras de Licenciatura en Genética.

\section{Consideraciones finales}

La hermenéutica es el "conjunto de conocimientos y técnicas que permiten que los signos hablen y nos descubran sus sentidos" (Foucault, 2010, p.47). La revisión conjunta de las resoluciones emitidas por CONEAU permite desglosar e identificar semejanzas y diferencias a fin de establecer cierto marco general de la situación de las carreras de Biotecnología y Genética en el contexto de la primera fase. Este trabajo nos permite ordenar y sistematizar la información que el proceso de acreditación logra hacer visible de manera fragmentada. En este sentido, las dimensiones de análisis elegidas permiten realizar un mapa general que sirve de referencia, tanto al interior de cada carrera como de cada disciplina en sí misma, y también permiten la comparación con otras acreditaciones en tanto siguen la perspectiva de análisis trazada por el Ministerio y la agencia evaluadora.

Estructurar nuestra exposición siguiendo la huella dejada por el Ministerio y CONEAU nos brindó la posibilidad de: 1) lograr un lenguaje común que facilite el análisis de los datos de las evaluaciones y que dialogue con el modelo de evaluación implementado, 2) consolidar los datos resultantes de la primera fase de acreditación para las disciplinas estudiadas, 3) identificar características disciplinares, fortalezas y desafíos, 4) contextualizar la situación de estas carreras al interior del sistema universitario a partir de la comparación con los datos resultantes de las primeras fases de otras disciplinas y 5) confeccionar un mapa de la acreditación de las carreras abordadas.

Conscientes de los límites del presente trabajo, consideramos que el próximo paso es recoger las perspectivas de actores claves sobre el proceso de acreditación como son las comunidades académicas de ambas disciplinas, teniendo en cuenta la tradición e impronta del sistema universitario argentino. Al avanzar sobre nuestra investigación fuimos trazando las pautas para nuestro próximo trabajo.

\section{Referencias bibliográficas}

Aiello, M. (2017). Repensando la evaluación y el aseguramiento de la calidad universitaria en la Argentina el Siglo XXI. En: La agenda universitaria III. Propuestas de políticas y acciones. Buenos Aires: Universidad de Palermo.

Araujo, S. (2007). Evaluación institucional y cambio universitario. Un difícil proceso de reconstrucción. En: Evaluando la evaluación. Políticas universitarias, instituciones y actores en Argentina y América Latina. Buenos Aires: Prometeo.

Araujo, S. y Trotta, L. (2011). La acreditación de las Ingenierías: configuración compleja en la institucionalización de la política. En: Revista Archivos de Ciencias de la Educación [en línea] Vol. 5, № 5. La Plata: UNLP. Recuperado de http://www.memoria.fahce.unlp.edu.ar/art_revistas/pr.5430/pr.5430.pdf

Banco Mundial (1997). Informe sobre Desarrollo Mundial 1997. El Estado en un Mundo en Transformación. Banco Mundial, Cap. 9.

Banco Mundial (1998). Más Allá del Consenso de Washington; la hora de la reforma institucional. Banco Mundial, Cap. 7.

Buchbinder, P. (2008). Masividad, heterogeneidad y fragmentación: el sistema universitario argentino 1983-2007. Buenos Aires: Biblioteca Nacional.

Buchbinder, P. (2010). Historia de las Universidades Argentinas. Buenos Aires: Sudamericana.

CINDA (2007). Informe sobre la Educación Superior en Iberoamérica. Santiago de Chile. Recuperado de: https://cinda.cl/publicacion/educacion-superior-en-iberoamerica-informe-2007/

Claverie, J.; González G. y Pérez, L. (2008). El sistema de evaluación de la calidad de la educación superior en la Argentina: el modelo de la CONEAU. Alcances y límites para pensar la mejora. En: Revista Iberoamericana de 
Evaluación Educativa [en línea] - Vol. 1, № 2. Recuperado de http://www.rinace.net/riee/numeros/vol1num2/art10.pdf

CONEAU (2014). Agronomía. Impacto en la calidad educativa. Buenos Aires: CONEAU. Recuperado de http://www.coneau.gob.ar/archivos/publicaciones/documentos/AcreditacionCarrerasGrado-Agronomia.pdf

CONEAU (2015). Ingeniería. Impacto en la calidad educativa. Buenos Aires: CONEAU. Recuperado de http://www.coneau.gob.ar/archivos/publicaciones/documentos/ingenieriaOk.pdf

CONEAU (2016). Veterinaria. Impacto en la calidad educativa. Buenos Aires: CONEAU. Recuperado de http://www.coneau.gob.ar/archivos/publicaciones/documentos/veterinaria2016.pdf

CONEAU (2017). Medicina. Impacto en la calidad educativa. Buenos Aires: CONEAU. Recuperado de http://www.coneau.gob.ar/archivos/publicaciones/documentos/Medicina2017.pdf

Corengia, A. (2015). El impacto de la CONEAU en las universidades argentinas. Buenos Aires: Editorial TeseoUniversidad Austral.

Fernández Lamarra, N. (2002). La Educación Superior en la Argentina. Buenos Aires: IESALC- UNESCO; SPU-MECyT Argentina. Recuperado de: http://unesdoc.unesco.org/images/0014/001494/149464so.pdf.

Fernández Lamarra, N. (2003). Evaluación y acreditación en la educación superior Argentina. Buenos Aires: IESALCUNESCO. Recuperado de https://eco.mdp.edu.ar/cendocu/repositorio/00104.pdf

Fernández Lamarra, N. (2007). Educación superior y calidad en América Latina y Argentina. Los procesos de evaluación y acreditación. Buenos Aires: IESALC/UNESCO. EDUNTREF.

Fernández Lamarra, N. (2010). La convergencia de la educación superior en América Latina y su articulación con los espacios europeo e iberoamericano: posibilidades y límites. En Revista da Avaliação da Educação Superior Campinas [en línea] - Vol. 15, №2. Recuperado de http://dx.doi.org/10.1590/s1414-40772010000200002

Fernández Lamarra, N. y Aiello, M. (2014). La evaluación universitaria en Argentina en el marco de América Latina. Situación y desafíos en relación al aseguramiento de la calidad. En: Monarca, H. (coord.) Evaluación de la calidad de la educación superior en Iberoamérica. España: UAM.

Fisher, M. (2018). Realismo capitalista. ¿No hay alternativa? Buenos Aires: Caja Negra.

Foucault, M. (2010). Las palabras y las cosas. Buenos Aires: Siglo XXI.

Gómez, J. (2016). Los mecanismos de evaluación institucional y acreditación de carreras y la calidad del sistema universitario argentino. En Debate Universitario - vol. 4, № 8. Buenos Aires: UAI.

Isuani, E. (2003). Estudio sobre algunos resultados de la labor de la CONEAU. Buenos Aires: CONEAU. Recuperado de http://www.coneau.gob.ar/archivos/1337.pdf

Krotsch, P. (1994). La emergencia del Estado Evaluador, el sistema universitario argentino y el surgimiento de la institución. En: La Universidad Hoy y Mañana. Perspectivas Latinoamericanas, J. E. Esquivel Larrondo (Coord.). Buenos Aires: ANUES.

Krotsch, P. (2001). Educación superior y reformas comparadas. Buenos Aires: UNQ editorial.

Krotsch, P. (2002). La Universidad Cautiva: marcas, legados y horizontes. La Plata: Editorial Al Margen.

Márquez, A. y Marquina, M. (S/D). Evaluación, Acreditación, Reconocimiento de Títulos, Acreditación. Enfoque comparado. Recuperado de https://web.fceia.unr.edu.ar/images/PDF/Evaluacion_acred.pdf

Naim, M. (1995). Latinoamérica: La segunda fase de la reforma. En: Revista Occidental, Estudios Latinoamericanos. 12, № 2. México.

Ozslak, O. (1999). De menor a mejor. El desafío de la segunda reforma del Estado. En: Revista Nueva Sociedad [en línea] $\quad N^{\circ} 160 . \quad$ Recuperado de http://www.campinas.sp.gov.br/arquivos/recursoshumanos/OSZLAKmenorymayor.pdf

Ozslak, O. (2005). Informe de consultoría en planeamiento de acciones de desarrollo institucional. Buenos Aires: CONEAU. Recuperado de http://www.coneau.gob.ar/archivos/internacionales/Oszlak.pdf

Robledo, M. del R. (2015). Aseguramiento de la Calidad de la Educación Superior. El caso de las agencias de acreditación en Chile y Argentina 1990-2010. V Congreso SAECE. Buenos Aires.

Síntesis de información de estadísticas universitarias: 2015-2016 (2016). Departamento de Información Universitaria, Secretaría de Políticas Universitarias (SPU). Buenos Aires: Ministerio de Educación.

Síntesis de información de estadísticas universitarias: 2016-2017 (2017). Departamento de Información Universitaria, Secretaría de Políticas Universitarias (SPU). Buenos Aires: Ministerio de Educación.

Villanueva, E. (2006). El sistema argentino de evaluación y acreditación universitaria [en línea] - Recuperado de https://eco.mdp.edu.ar/cendocu/repositorio/00080.pdf

\section{Marco normativo y fuentes}

Ley № 24.521 de Educación Superior. Congreso Nacional de Argentina, 7 de agosto de 1995.

Decreto N²68/95. Poder Ejecutivo Nacional, 7 de agosto de 1995.

Decreto $N^{\circ}$ 499/95. Poder Ejecutivo Nacional, 22 de septiembre de 1995.

Decreto $N^{\circ} 173 / 96$. Poder Ejecutivo Nacional, 21 de febrero de 1996.

Decreto $N^{\circ}$ 705/97. Poder Ejecutivo Nacional, 30 de julio de 1997.

Decreto N ${ }^{\circ} 2219 / 10$. Poder Ejecutivo Nacional, 30 de diciembre de 2010.

Resolución Ministerial №6/97. Ministerio de Educación, 13 de enero de 1997.

Resolución Ministerial №51/10. Ministerio de Educación, 2 de febrero de 2010.

Resolución Ministerial № 244/16. Ministerio de Educación y Deportes, 28 de abril de 2016.

Resolución Ministerial № 280/16. Ministerio de Educación y Deportes, 29 de abril de 2016.

Resolución Ministerial № 284/16. Ministerio de Educación y Deportes, 3 de mayo de 2016.

Resolución Ministerial № 1637/17. Ministerio de Educación y Deportes, 4 de abril de 2017.

Resolución Ministerial № 901/17. Ministerio de Educación y Deportes, 6 de marzo de 2017.

Resolución Ministerial № 903/17. Ministerio de Educación y Deportes, 6 de marzo de 2017

Acuerdo Plenario $\mathrm{N}^{\circ}$ 137/15. Consejo de Universidades, 20 de octubre de 2015.

Acuerdo Plenario $\mathrm{N}^{\circ}$ 138/15. Consejo de Universidades, 20 de octubre de 2015.

Acuerdo Plenario $\mathrm{N}^{\circ}$ 142/16. Consejo de Universidades, 9 de noviembre de 2016.

Acuerdo Plenario № 143/16. Consejo de Universidades, 9 de noviembre de 2016.

Acuerdo Plenario $N^{\circ}$ 144/16. Consejo de Universidades, 9 de noviembre de 2016.

Ordenanza CONEAU No 12/97. CONEAU, 9 de septiembre de 1997.

Ordenanza CONEAU № 63/17. CONEAU, 14 de diciembre de 2017.

Ordenanza CONEAU No 70/20. CONEAU, 4 de agosto de 2020. 
Acta CONEAU No 481/18. CONEAU, 7 de mayo de 2018 .

RESFC-2017-494-APN-CONEAU\#ME. CONEAU, 16 de noviembre de 2017.

RESFC-2017-495-APN-CONEAU\#ME. CONEAU, 16 de noviembre de 2017.

RESFC-2017-570-APN-CONEAU\#ME. CONEAU, 18 de diciembre de 2017.

RESFC-2017-569-APN-CONEAU\#ME. CONEAU, 18 de diciembre de 2017.

RESFC-2019-166-APN-CONEAU\#MECCYT. CONEAU, 27 de mayo de 2019.

RESFC-2019-287-APN-CONEAU\#MECCYT. CONEAU, 16 de julio de 2019.

RESFC-2019-176-APN-CONEAU\#MECCYT. CONEAU, 27 de mayo de 2019.

RESFC-2019-290-APN-CONEAU\#MECCYT. CONEAU, 16 de julio de 2019.

RESFC-2020-141-APN-CONEAU\#ME. CONEAU, 9 de junio de 2020.

RESFC-2019-175-APN-CONEAU\#MECCYT. CONEAU, 27 de mayo de 2019.

RESFC-2020-167-APN-CONEAU\#ME. CONEAU, 6 de julio de 2020.

RESFC-2019-177-APN-CONEAU\#MECCYT. CONEAU, 27 de mayo de 2019.

RESFC-2019-280-APN-CONEAU\#MECCYT. CONEAU, 16 de julio de 2019.

RESFC-2020-70-APN-CONEAU\#ME. CONEAU, 4 de mayo de 2020.

RESFC-2019-165-APN-CONEAU\#MECCYT. CONEAU, 27 de mayo de 2019.

RESFC-2019-533-APN-CONEAU\#MECCYT. CONEAU, 13 de noviembre de 2019.

RESFC-2019-164-APN-CONEAU\#MECCYT. CONEAU, 27 de mayo de 2019.

RESFC-2019-163-APN-CONEAU\#MECCYT. CONEAU, 27 de mayo de 2019.

RESFC-2019-302-APN-CONEAU\#MECCYT. CONEAU, 16 de julio de 2019. 